\title{
QM nicht geeignet für Medizin
}

\author{
Warum Qualitätsmanager sich nicht in Frage stellen lassen
}

Seit nun bald Jahrzehnten wird die Medizin von Nichtmedizinern mit angeblichen qualitätserhöhenden Methoden "gequält". Obwohl faktisch keine Evidence based-Daten existieren, dass Total Quality Management (TQM)-Systeme einen Nutzen bringen, haben viele hochkarätige Ärzte diese Methoden (EFQM, ISO, DMP, BENCHMARKING etc.) unkritisch übernommen und bemühen sich, damit Qualität zu verbessern. Dabei können schon theoretische Überlegungen klar machen, dass diese TQMs nicht das leisten können, was sie vorgeben.

\section{Wer bestimmt, was in der Medizin Qualität ist?}

$\mathrm{Zu}$ allererst der Patient. Er wird dem Arzt nach abgeschlossener Behandlung mitteilen, ob er zufrieden war. Allerdings ist das nicht sicher. Aus Evidence based-Daten wissen wir, dass auch nach standardisierter Methode der Aufklärung, die Patienten in der überwiegenden Zahl nachher nicht wissen, was mit ihnen geschieht. Der Patient, der noch nie eine Operation hatte, kann sich aus rein rhetorischer Sicht keine Vorstellung darüber machen, was mit ihm passiert. Ärzte haben eine zumindest zehnjährige Ausbildung, um sich zu erklären, was sie denn tun. Dies ist auch noch für jeden Arzt verschieden. Dazu kommt eine mit jeder Krankheit einhergehende Angst (muss ich sterben?), die einen erheblichen Stress darstellt und die Informationsverarbeitung beeinträchtigt.

Da jeder Patient und jede Situation verschieden ist, gibt es keine objektiv richtige verbale Methode zur medizinischen Aufklärung. Denn selbst wenn der Patient versteht, was geschieht, bleibt noch immer das Erleiden und das Erleben des Erleidens ist nicht vorhersagbar. Echtheit lässt sich im Leben oft nur schwer kommunizieren. Ob jemand authentisch ist, ob er das meint, was er sagt, ob er das versteht, was er sagt, lässt sich nicht letztgültig veri-

\footnotetext{
1 Univ.-Prof. Dr. Kaspar Sertl, Systemischer Organisationsberater, Gruppendynamiktrainer, Primarius, Pianist, Wien
}

fizieren und vor allem besteht immer die Möglichkeit, dass das Ergebnis ganz anders ist als erwartet - positiv wie negativ.

\section{Eine Frage des Blickwinkels}

Ob etwas gut oder schlecht ist, hängt vom Blickpunkt und den Interessen des Beobachters ab. Qualitätsmanager werden immer Verbesserungsmöglichkeiten finden, weil sie davon leben. Patienten sind meist in alle Richtungen offen, die Einschätzung kann gut oder schlecht sein - unabhängig vom Ergebnis, aber sehr abhängig von seinen Vorabwünschen. Ärzte werden ihre medizinischen Werte als Kriterien heranziehen („she dies, but balanced“), Juristen, die ja - medizinische Laien sind, werden gerne Kommunikation heranziehen, weil diese nicht objektivierbar ist und man damit auch Prozesse gewinnen kann. Politiker schmücken sich gerne damit, dass sie ein Kontrollsystem eingeführt haben, das nun alle Fehler ausschließt - auch wenn die Kosten explodieren (siehe ELGA).

\section{Die Aussagekraft von Checklisten}

Wie soll nun ein den medizinischen Inhalt reduzierendes System wie EFQM oder ISO in der Lage sein, diese vielen Befindlichkeiten auf einen Nenner zu bringen? Dazu müsste man vorher wissen, was sich die einzelnen Teilnehmer erwarten. Beim Vergleich mit dem Resultat ergibt sich die Schwierigkeit festzustellen, ob dieses nun nicht/oder doch so ist, wie es sich der Patient vorher vorgestellt hat. Menschen benutzen gleiche Worte und meinen Verschiedenes und umgekehrt: Menschen benutzen verschiedene Worte und meinen das Gleiche.

Qualitätssysteme, die letzendlich alle auf Checklisten reduziert sind, können freilich nicht feststellen, ob das eingetreten ist, was vorher festgelegt wurde. Auf der Grundlage von Checklisten sind bereits Flugzeuge abgestürzt. Checklisten werden abgehakt, auch wenn die angekreuzten Dinge nicht durchgeführt wurden. Für Patienten sind abgehakte Checklisten völlig irrelevant.

\section{Wiederaufnahmen und Notfallambulanz}

Kontrollierte Studien stellen fest, dass so wichtige Kriterien wie Wiederaufnahmen und Besuch von Notfallambulanzen durch diese Systeme nicht reduziert werden können. Zieht man diese beiden Komponenten als Ergebnis-Messung heran, kommt es zu keiner Verbesserung. Die Frage bleibt: Wieso soll es ein schlechtes Qualitätskriterium sein, wenn jemand ins Spital kommt oder in die Notfallambulanz? Aus medizinischer Sicht ist dies ja keine schlechte Qualität (übrigens evidence based) aber angeblich aus ökonomischer Sicht. Evidence based zeigt sich aber, dass eine zu lange ambulante Behandlung zu Kostenexplosionen führen „Wir sparen, koste es, was es wolle“.

TQM-Systeme - und zwar offenbar alle - bringen keine Sicherheit, erhöhen keine medizinische Qualität, senken keine Kosten. Die Frage bleibt: Warum machen wir sie weiter? Dass Qualitätsmanager sie nicht aufgeben wollen, ist klar, weil sie davon leben, diese zu implementieren und zu betreuen. Sie gehen auch soweit, in ihren Zeitungen zu verhindern, dass kritische Artikel zu QM publiziert werden. Sie fordern dann auch gerne von den Ärzten ein, evidence-basiert zu agieren.

Generaldirektoren von Krankenkassen geben dabei gerne $\mathrm{zu}$, „dass die Systeme nichts bringen, aber wir haben nichts anderes". Leider haben diese Personen in ihrem Gedankenrepertoire nicht die Variante: „Wir beenden es, weil es teuer ist und nichts bringt und sparen uns viel Geld." Würden sie nur einmal bei der Personalausstattung so denken! (Z. B. Diabetiker gehen nicht zum Augenarzt weil es vier bis sechs Monate dauert, einen Termin zu bekommen und man dann vier Stunden dort sitzt.) Wir würden viel Sicherheit und Qualität gewinnen - ohne TQMs.

Korrespondenz:

Univ.-Prof. Dr. Kaspar Sertl

E-Mail: kaspar.sert!@wienkav.at 